\title{
DETERMINING THE TIME CONSTANT OF ARCS AT ARBITRARY CURRENT LEVELS
}

\author{
L. S. J. BorT*, T. Schultz, C. M. Franck \\ High Voltage Laboratory, ETH Zurich, Physikstrasse 3, 8092, Zurich, Switzerland \\ * lorenz.bort@alumni.ethz.ch
}

\begin{abstract}
.
For the development and optimization of gas circuit breakers and switchgear, a detailed understanding of the arc related processes is of great importance. Ideally, analytical or numerical models with predicitive capability can be found and used during the design process preceding costly and time-consuming experiments.

In the present contribution, we report on a novel measurement and evaluation technique to determine the thermal arc time constant ("thermal inertia") that is commonly used in simple black-box models to describe the arc's dynamical properties. The method is introduced and applied to example arcs under varying blow gas conditions in air.
\end{abstract}

Keywords: switching arc, gas circuit breakers, black-box model, arc time constant.

\section{Introduction}

The understanding of electric arcs is crucial for the design and improvement of mechanical switches in the power system. This applies for a wide range of switches, from low voltage AC to HVDC. One important internal parameter of arcs is their time constant. It describes a characteristic time for the exchange of energy between arcs and their surrounding. This has a considerable impact on the electrical behavior of the arc.

It is well established that interruption becomes more difficult for higher current gradients before zero crossing. This is due to the fact, that the conductance of the arc at zero crossing is not at its steady state for zero current, i.e. zero, but at a finite value. For current zero crossing, this means that the arc still contains considerable thermal energy and thus still conducts current. The conductance at current zero (CZ) depends on the arc time constant, as well as on the current gradient before CZ. For steep current gradients, or large time constants, the conductance at current zero is high, which can lead to reignitions. As the time constant varies with gas flow conditions, nozzles and gas types, it is one important performance parameter of mechanical interrupters.

In HVDC, current interruption can be realized using a mechanical circuit breaker and a counter current injection circuit. In these current injection topologies, the current wave shape (especially at $\mathrm{CZ}$ ) is determined by the circuit breaker itself. Consequently, it is possible to implement circuits that drastically decrease the current gradient shortly before CZ to improve interruption $[1,2]$. The length of the phase with decreased current gradient needed to improve interruption performance is defined by the arc time constant in this case. The arc time constant is thus an optimization parameter.
Another field of application where it is important to know the arc time constant is the optimization of passive oscillation switches, which are commonly used as transfer switches (most notably the metal return transfer switch) in HVDC applications. Here, the voltage-current characteristic of interrupters leads to the growth of an oscillating current. For fast interruption, high current oscillation frequency is helpful, as long as the the oscillation period is longer than $20 \tau$. This means in this application, the arc time constant of the should be minimized.

To determine the characteristic time constant $\tau$ of a given setup, several possibilities exist [3-7], which were evaluated in previous work $[8,9]$. All of these have certain drawbacks, and do not allow the determination of thermal inertia at arbitrary current levels.

According to $[8,9]$ a staircase current waveform would be ideal, but short current impulses superimposed to quasi-DC current are proposed if a step with sufficiently steep slope is impossible to create. In this paper, a method to determine the time constant of arcs, based on triangular impulse currents, is developed and implemented. As validation case, tests showing the transition between free-burning and blown arc are presented.

\section{Theory}

The response of an arc to dynamic changes in current is generally described by black box models, namely the ones of Mayr and Cassie [10, 11]. These models assume that the loss terms are a function of the arc conductance $g$ and cannot change instantaneously. Some time is needed to change to the new equilibrium state, which can be described by a time constant. In case of a current increase from $i_{0}$ to $i_{1}$, it must be a change that leads to a greater conductance $g_{1}$. This change requires a certain change of the arc enthalpy, 
to either increase the arc radius, or the temperature. The required energy has to be supplied by the difference of the instantaneous power the arc loses to its surroundings $P_{\text {cool }}$ (due to radiation, convection and thermal conduction) and instantaneous heating power $u \cdot i$. The Mayr model is usually used to describe arcs during a few microseconds before and after current zero in AC circuit breakers. It assumes the instantaneous cooling power $P_{\text {cool }}$ is constant and there is one time constant $\tau$ which describes the relaxation process. Cassie, Habedank, Shavemaker and many others [11-13] generalized this, by making $P_{\text {cool }}$ and possibly $\tau$ a function of $g$, to describe the high current phase as well. The most general form is therefore

$$
\frac{1}{g} \frac{d g}{d t}=\frac{1}{\tau(g)}\left(\frac{u i}{P_{\mathrm{cool}}(g)}-1\right) .
$$

The physical interpretation of this model is that the arc consists of one energy reservoir (the enthalpy of the heated plasma column) and that the conductance is the fundamental variable to describe its state. In steady state, ohmic heating equals the sum of all cooling terms, i.e. $P_{\text {cool }}=u \cdot i$, and therefore $g$ (and with it the arc enthalpy) doesn't change. In transient conditions, for example at falling current, there is a difference between heating and cooling power, leading to heating or cooling of the plasma, i.e. the arc adapts to changing conditions by shrinking for example. For simplicity, the most general form of the transient arc model equation (1) will be called Mayr Equation throughout the manuscript, although Cassie Equation would be equally right or wrong, because the two only differ in how $P_{\text {cool }}$ depends on $g$ (see [14-16]).

The literature mentioned above provides different modified models, which assume certain analytic functions for $P_{\text {cool }}(g)$ and $\tau(g)$. Cassie for example assumes a constant voltage during high currents. Schwarz [3] introduced some additional variables to parameterize $P_{\text {cool }}(g)$ and $\tau(g)$, and more recently also other modifications were published. A summary of several models can be found in [16]. In the present work, those more specific models were not studied, but the generalized form was used. The only constraint this general form has is that both $P_{\text {cool }}(g)$ and $\tau(g)$ only depend on $g$ and not on $u$ or $i$, without specifying a priori what that dependence should be. Other external factors, like contact separation distance and blowing gas flow obviously also impact the arc. The Mayr equation (1) assumes those external conditions remain constant.

This arc model leads to the following behaviour: All changes of external conditions - namely how much current flows through the arc - that take much longer than $5 \tau$ can be considered quasi-static. The difference between $P_{\text {cool }}$ and $u \cdot i$ will always be virtually zero, and the system will be in equilibrium any time.

On the other hand, current variations that happen on a time scale much shorter than $\tau$ do not allow the arc to react. The conductance will not change, and

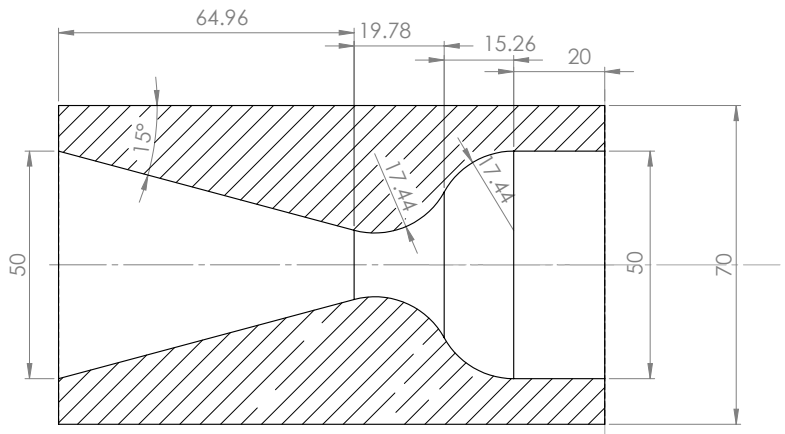

Figure 1. Nozzle used for the experiments, made from PMMA to allow optical observation. Gas flow is from right to left. The downstream contact tip is flush with the nozzle exit plane, the upstream contact protrudes a few $\mathrm{mm}$ into the nozzle segment that is still straight.

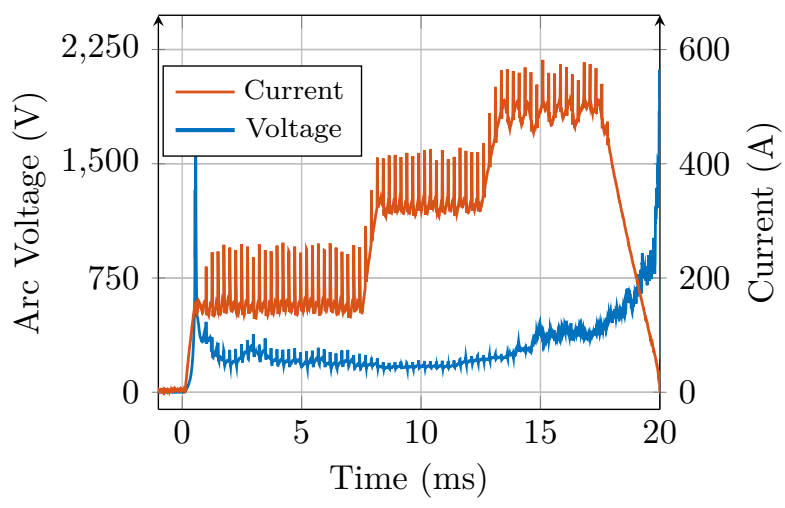

Figure 2. Current and arc voltage during measurement to determine arc constant $\tau$ at three current levels.

the arc will behave like a resistor [10]. Any method that aims to determine the arc time constant should create current changes on a time scale that is between these two extreme regions.

\section{Experimental Methods}

The DUT is a model interrupter using air, described in more detail in [2]. A converging-diverging nozzle with single flow direction is used, as shown in figure 1 . The contacts are stationary and have a diameter of $22 \mathrm{~mm}$. The arc is initiated using an ignition wire. The blowing system uses pressure stored in gas bottles and fast acting valves.

A flexible pulsed direct current source (FPDCS) $[9,17]$ is used to generate the current waveforms for testing. The current shape can be formed by up to three independently acting buck-converter modules.

In the performed tests, a base current level was kept by a module with large inductance $(4.5 \mathrm{mH})$. On this, fast rising spikes are superimposed by a second module with low inductance $(0.2 \mathrm{mH})$. The voltage and current traces for a measurement with three different base current levels are illustrated in Figure 2. 


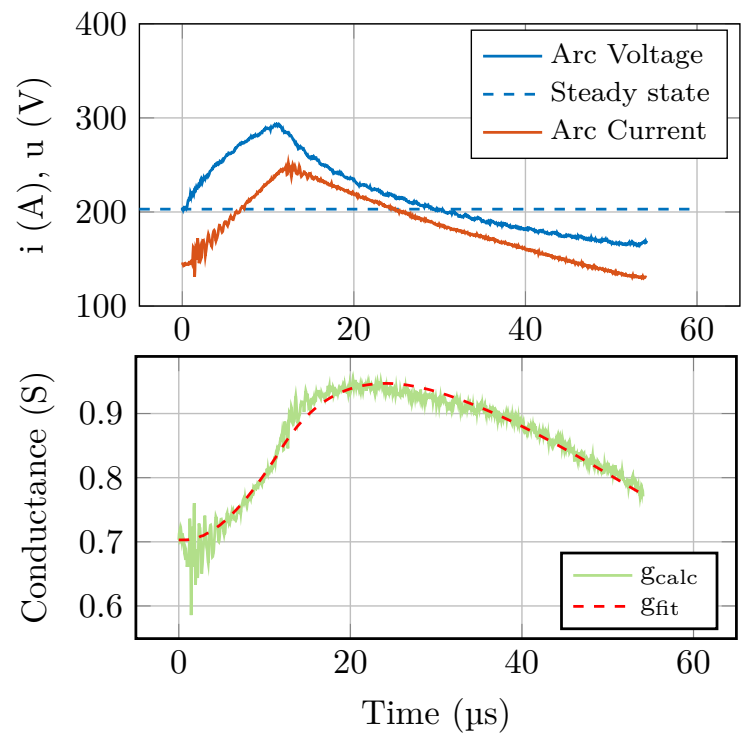

Figure 3. Close-up of a single spike, used to determine arc time constant $\tau$. Top plot shows measured values $u$ and $i$, bottom plot shows $g_{\text {calc }}=i / u$ and the fit for $\tau=$ $36 \mu s, P_{\text {cool }, 1}(0.71 S)=29 k W$ and $P_{\text {cool }, 2}(0.95 S)=$ $45 k W$.

Figure 3 shows measured voltage and current for a representative single spike. The conductance calculated from voltage and current, as well as the fit by the described algorithm are illustrated as well.

\section{Fitting Algorithm}

Determination of $\tau$ means finding a solution for which equation (1) accurately reproduces the measured results, without using any analytical formula that expresses $\tau$ as a function of other parameters. This requires experimental data of dynamic arc behavior, i.e. current and voltage waveforms of an arc that is perturbed from a steady state. In the present study, a quasi-static DC current (gradient lower than $1 \mathrm{~A} \mathrm{us}^{-1}$ ) is superimposed with transient current impulses of triangular wave-shape of high gradient (greater then $10 \mathrm{~A} \mathrm{ps}^{-1}$ ), as shown in Figure 2. If the rise time and fall time of the impulse are in the order of magnitude of $\tau$, it is possible to determine $\tau$, by a method proposed in [9].

First, a value of $\tau$ is assumed. Then, the conductance and voltage response of an arc with the assumed value of $\tau$ is calculated with equation (1). In a third step, the calculated conductance waveform is compared to the measured values $i / u$, by calculating the root mean square difference. This process is repeated for different values of $\tau$, until a minimum difference is found.

In the present work, an algorithm to solve the Mayr equation was implemented, tested and used to perform the fit. A key requirement to the algorithm is to avoid the need to calculate derivatives of measured signals. Differentiating measured current and voltage signals accurately and with sufficient bandwidth is impossible, due to the inherent decrease of signal to noise ratio of derivatives. The optimized fitting algorithm instead uses the forward Euler method to solve equation (1), which makes the following assumptions:

$\square$ The arc is in steady state before the current transient, therefore all variables at $t_{0}$ are known $g_{0}=$ $i_{0} / u_{0}, P_{\text {cool }, 0}\left(g_{0}\right)=u_{0} i_{0}$

$\square$ During the current transient changes in $g$ are small enough so $\tau(g)$ can be considered constant.

$\square$ During the current transient, $P_{\text {cool }}(g)$ can be linearized, by specifying a second point $P_{\text {cool, } 1}\left(g_{1}\right)$ additional to the starting point $P_{\text {cool }, 0}\left(g_{0}\right)$.

$\square$ There are no random fluctuations during the transient period due to other reasons that would also alter $P_{\text {cool }}(g)$, like arc root movement etc.

With these assumptions, initial conditions and assumed values for $\tau$ and $P_{\mathrm{cool}, 1}\left(g_{1}\right)$, equation (1) can be used to solve for the conductance waveform $g(t)$, if the current waveform $i(t)$ is given. This is done by iteratively calculating $\dot{g}(t), g(t)$ and $u(t)$ for one point in time, using the values from the previous time step. To minimize the error, time steps much smaller than $\tau$ have to be used. Since the sample rate of current and voltage is high (>50 MS) to reject common mode noise of the source (see [17]), this is not a problem.

The result for idealized current waveforms is shown in Figure 4. The response of the arc depends on the slope and duration of the current spikes, relative to $\tau$. For the first spike $\tau$ was set to $1 / 4$ of the rise time, which means the conductance lags behind the instantaneous value, but has a similar shape. For the second spike $\tau$ is equal to the rise time. Here, the lag of the conductance is so significant that the shape of the waveform starts to differ from the hypothetical steady state curve. This also leads to bigger changes of the arc voltage. The last spike has a $\tau$ four times longer than the rise time, meaning the current rises and falls so fast that the conductance can hardly follow. Peak conductance is reached almost at the end of the spike, and afterwards exponential decay follows.

To aid interpretation, dashed lines show the voltage and conductance values one would obtain for an arc that is always in steady state, i.e. for $\tau=0$. Dotted lines represent the resistive case, in which the arc conductance does not change at all, i.e. for $\tau=\infty$. In this picture it becomes clear why a rise time around $\tau$ is favoured. For this spike, the conductance changes significantly, but at the same time it is also significantly delayed compared to the steady curve. The voltage curve is in between the two extremes during the rising edge, and shows significant undershoot on the falling edge. The left spike with rise time $4 \tau$ is too slow for the arc to be transient. Its conductance follows the steady state curve closely, which leads only to a small deviation of the voltage from the static $u(i)$ value. This makes it hard to determine a numeric value for $\tau$, especially if the deviation of the voltage 

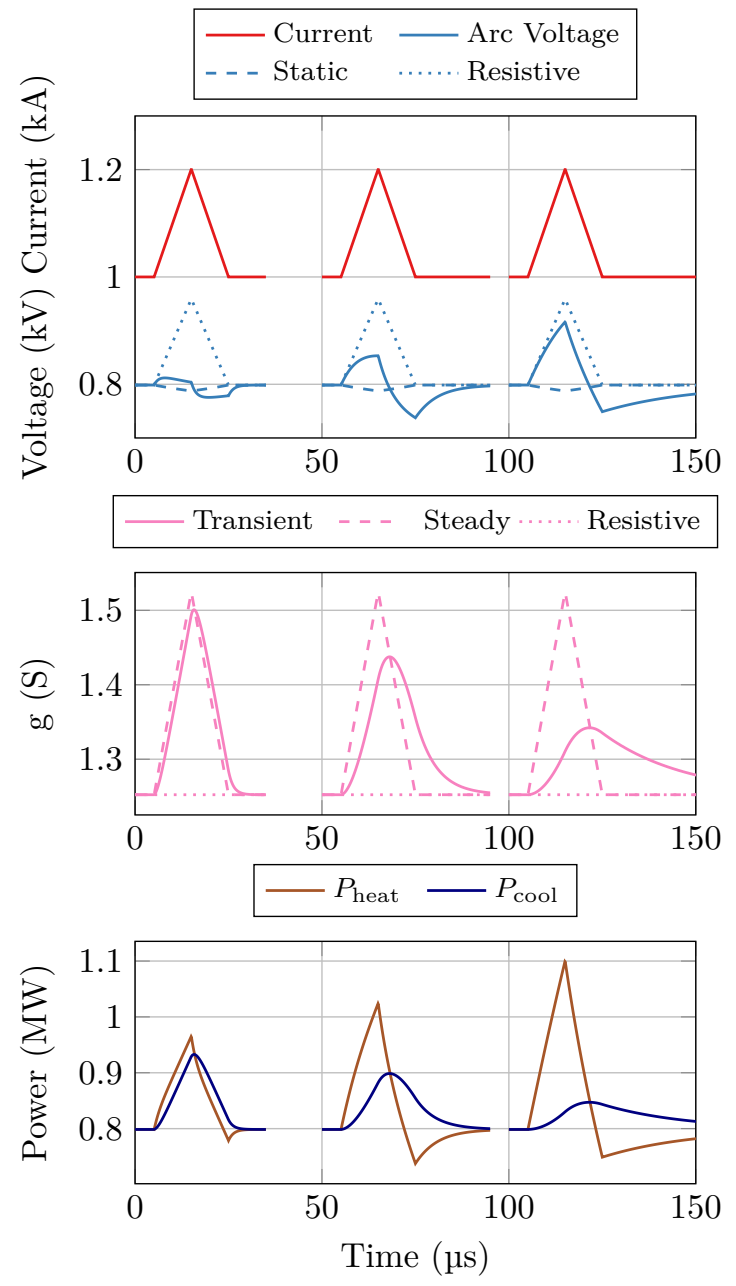

Figure 4. Simulated response of an arc to triangular current impulses with $10 \mu \mathrm{s}$ rise and fall time. Arc time constant $\tau$ is changed for each spike (2.5/10/40 $\mu$ s).

curve becomes small enough to be in the order of the inductive voltage drop between the two voltage probes. The rightmost spike is too fast for the arc to properly react at all. Its conductance does not change as much, and the voltage follows the resistive curve.

\section{Results}

To test the sensitivity of the presented method, several measurements have been conducted and analyzed.

To introduce a considerable change in the arc's thermal inertia, in the first experiment the transition between free burning and blown conditions at fixed base current level are observed. Figure 5 illustrates pressure build-up in the nozzle throat, arc voltage and current as well as $\tau$ for this measurement. Compared to the blown phase, cooling is considerably lower in the free burning phase. As expected, this manifests in a lower arc voltage. Optical investigations with a high speed camera show a large luminescent region. When gas flow is initiated at around $15 \mathrm{~ms}$, this region shrinks which coincides with a decreasing time constant, even before there is significant pressure change at the throat. As pressure builds up, the arc voltage
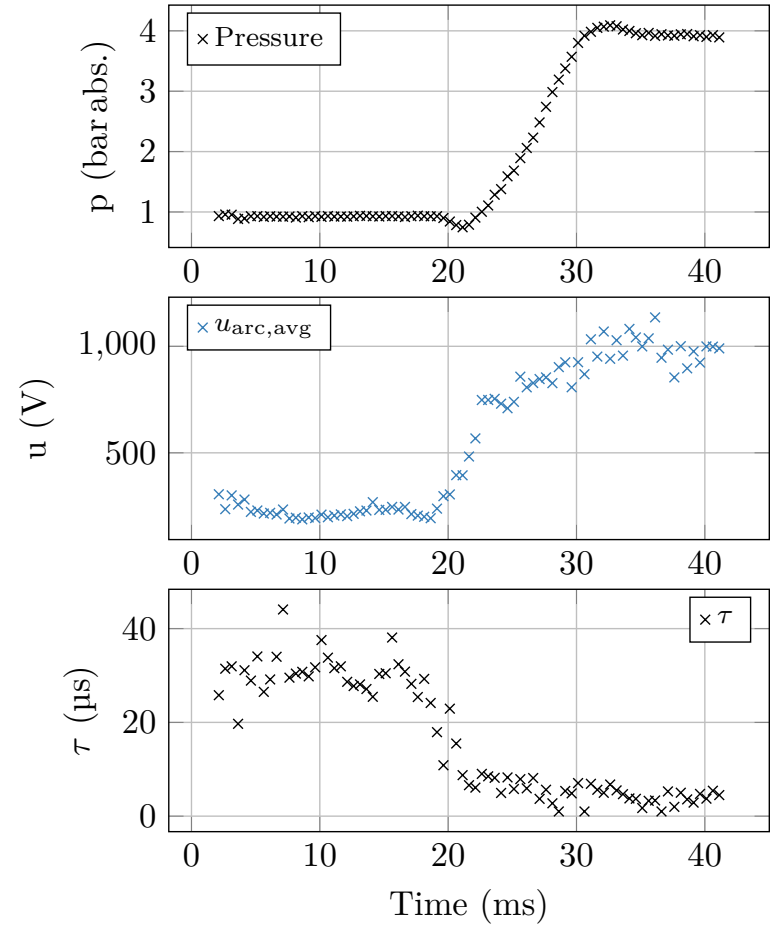

Figure 5. $\tau$ for variable pressure for a base current of $130 \mathrm{~A}$ and a spike amplitude of $100 \mathrm{~A}$. Gasflow starts accellerating around $16 \mathrm{~ms}$ as seen by highspeed footage.

increases until the pressure reached its new steady state value.

The development of $\tau$ is reasonable. A smaller, more confined arc is able to exchange energy with its surrounding faster, resulting in a reduced thermal inertia. Arc radius as well as measured $\tau$ start to decrease considerably as soon as gas flow is observed on the highspeed video, which is already a few ms before the flow chockes and pressure build-up is measured, as shown in figure 6 . Consequently, the measurements can be seen as a proof of concept for the presented method.

In a second step, blowing pressure is kept constant and the arc's time constant is evaluated for a fixed current level. The results for 115 spikes are illustrated in in Figure 7. It can be seen that the arc time constant under these conditions is mainly around $\tau=5 \mu \mathrm{s}$ with values ranging from $\tau=0 \mu \mathrm{s} \ldots 10 \mu \mathrm{s}$. Without further investigations it cannot be concluded if this spread represents real variations in arc time constants or the uncertainty of the method itself.

\section{Conclusions}

An important characteristic of (switching) arcs is the time constant with which the arc can exchange thermal energy with its surrounding and therefore change its conductance.

For this article, a method to determine thermal inertia (i.e. time constant) of arcs by using triangular impulse currents has been implemented. In example 


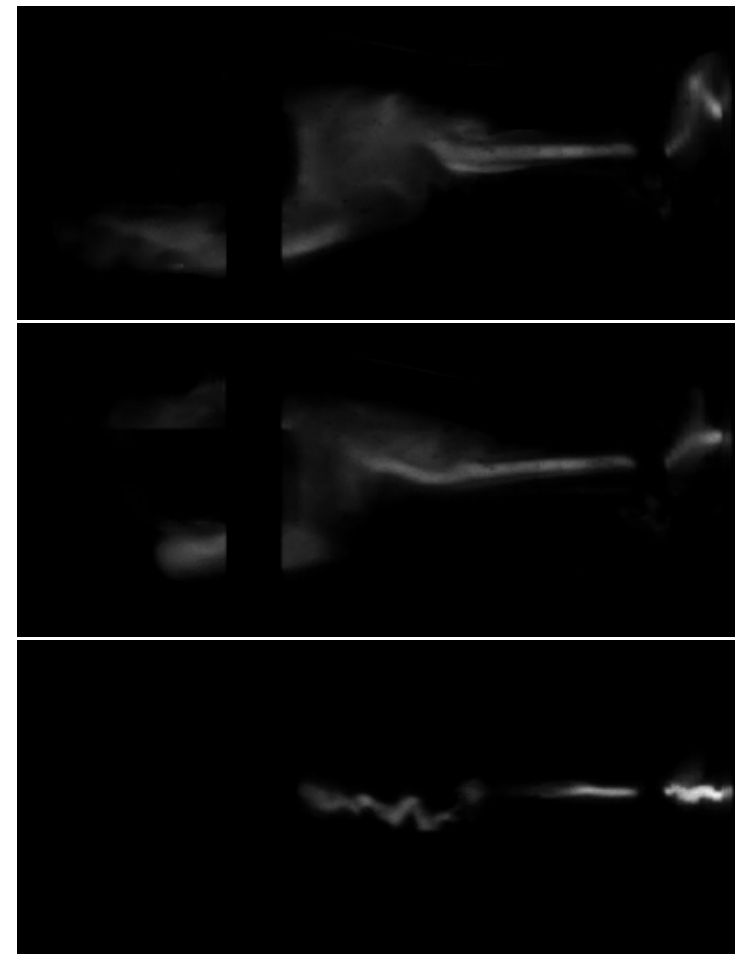

Figure 6. Three frames form highspeed video, showing appoximately the same part as figure 1. Top: Freeburning stage, captured at 13.120 ms, Center: transition, flow already visible upstream arc starts contracting, captured at 19.125 ms. Bottom: Chocked flow conditions reached, arc radius fully contracted, caputured at 24.620 ms. Exposure time was $0.16 \mu s$ in all cases.

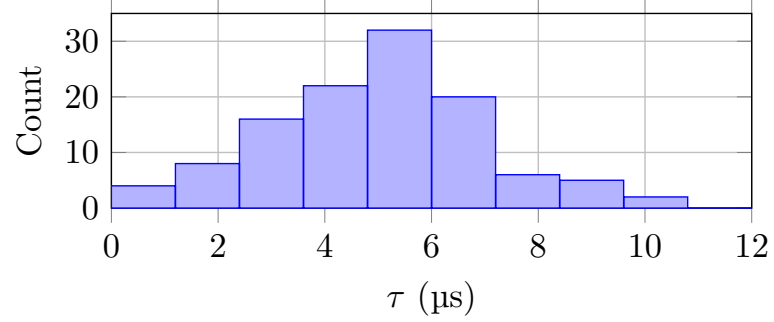

Figure 7. Histogram of the fitted arc time constant $\tau$ for a base current of $140 \mathrm{~A}\left(N_{\text {total }}=115\right.$ spikes $)$.

measurements, thermal inertia of an axially blown switching arc has been investigated for the transition from free-burning to blown. The results align well with the expected development, which makes this method a promising tool for future investigations. The logical next improvement of the method is a more detailed analysis of the scattering of $\tau$ shown in figure 7 , especially to distinguish between real arc properties and experimental uncertainty.

\section{Acknowledgements}

The authors would like to thank ABB Switzerland for financial support, and the master student Benjamin Hammerich for his help during experiments. This research is part of the activities of the Swiss Centre for Competence in Energy Research on the Future Swiss Electrical Infrastructure (SCCER-FURIES).

\section{References}

[1] A. N. Greenwood and T. H. Lee. Theory and application of the commutation principle for hvdc circuit breakers. IEEE PAS, PAS-91(4):1570-1574, July 1972. doi:10.1109/TPAS.1972.293310.

[2] T. Schultz et al. Improving interruption performance of mechanical circuit breakers by controlling pre-current-zero wave shape. IET High Voltage, pages 1-9, apr 2019. doi:10.1049/hve.2018.5103.

[3] J. Schwarz. Berechnung von Schaltvorgängen mit einer zweifach modifizierten Mayr-Gleichung. Dissertation, Technische Hochschule Darmstadt, 1973.

[4] R. Amsinck. Verfahren zur Ermittlung des Ausschaltverhalten bestimmender Lichtbogenkenngrössen. ETZ-A, 98:566, 1977.

[5] H. Rijanto. Ein experimentelles Verfahren zur Bestimmung von Lichtbogenkenngrößen -

Stromüberlagerungsverfahren. Dissertation, Technische Universität Hannover, 1975.

[6] R. Ruppe. Experimentelle und theoretische Untersuchungen am axial beströmten Wechselstromlichtbogen vor dem Stromnulldurchgang. Dissertation, TH Illmenau, 1979.

[7] H. Drebenstedt et al. Ein verbessertes Verfahren zur Bestimmung der charakteristischen Funktionen des Zweipolmodells für Schaltlichtbögen. Internat. Wiss. Kolloquium, TH Ilmenau, 28, 1983.

[8] M. M. Walter, C. Leu, and C. M. Franck. Optimizing the Arc Characteristics for Improved Designs of Passive Resonant Hvdc-Circuit Breakers. In XXth Symposium on Physics of Switching Arc, pages 317-322, 2013. doi:10.3929/ethz-b-000078010.

[9] M. M. Walter. Switching Arcs in Passive Resonance HVDC Circuit Breakers. PhD thesis, ETH Zürich, 2013 doi:10.3929/ethz-a-010112102.

[10] O. Mayr. Beiträge zur Theorie des statischen und des dynamischen Lichtbogens. Archiv für Elektrotechnik, 37(12):588-608, dec 1943. doi:10.1007/BF02084317.

[11] A. N. Cassie. Arc rupture and circuit severity: a new theory. CIGRE-Report, 102:1-16, 1939.

[12] U. Habedank. Application of a new arc model for the evaluation of short-circuit breaking tests. IEEE PWRD, 8(4):1921-1925, 1993. doi:10.1109/61.248303.

[13] P. H. Schavemaker and L. van der Sluis. An improved Mayr-type arc model based on current-zero measurements [circuit breakers]. IEEE PWRD, 15(2):580-584, apr 2000. doi:10.1109/61.852988.

[14] M. Kapetanović. High voltage circuit breakers. ETF Faculty of Eletrotechnical Engineering, Sarajevo, Sarajevo, 2011.

[15] K. Nakanishi. Switching Phenomena in High-Voltage Circuit Breakers. Marcel Dekker Inc., New York, NY, 1 edition, 1991.

[16] A. Khakpour et al. An Improved Arc Model Based on the Arc Diameter. IEEE PWRD, 31(3):1335-1341, jun 2016. doi:10.1109/TPWRD.2015.2473677.

[17] A. Ritter, L. S. J. Bort, and C. M. Franck. Five years of pulsed current testing for HVDC switchgear. In 2016 IEEE ICHVE Conference, pages 1-4. IEEE, sep 2016. doi:10.1109/ICHVE. 2016.7800658. 